\title{
Gate Optimization Analysis of Injection Molding for Mobile Phone Panel Based on Moldflow and Drop Simulation
}

\author{
Hongbing Wang ${ }^{1,2}$, Chunhua Sun ${ }^{1,2}$ \\ ${ }^{1}$ Department of Mechanical \& Electrical Engineering, Suzhou Vocational University, Suzhou, China \\ 23C-Product Intelligent Manufacturing Engineering Technology Research and Development Center of Jiangsu Province, Suzhou, China \\ Email: whb@jssvc.edu.cn
}

How to cite this paper: Wang, H.B. and Sun, C.H. (2021) Gate Optimization Analysis of Injection Molding for Mobile Phone Panel Based on Moldflow and Drop Simulation. World Journal of Engineering and Technology, 9, 92-99.

https://doi.org/10.4236/wjet.2021.91007

Received: January 8, 2021

Accepted: February 2, 2021

Published: February 5, 2021

Copyright (c) 2021 by author(s) and Scientific Research Publishing Inc. This work is licensed under the Creative Commons Attribution International License (CC BY 4.0).

http://creativecommons.org/licenses/by/4.0/

\begin{abstract}
Aiming at the gate design problem of a mobile phone panel, the plastic forming analysis software Moldflow and drop simulation were used to optimize the gate design. Six different gate design schemes were compared and studied, and the weld lines positions and injection molding results under each gate scheme were analyzed. Through the simulation analysis of the falling process of the mobile phone panel by the display dynamics analysis software ANSYS Workbench LS-DYNA, five analysis schemes were set according to the falling angle, and the stress distribution at the weld lines positions of the mobile phone panel in each scheme was studied. The reasonable gate scheme was given by synthesizing the force condition, the mold flow analysis and the drop simulation results of the mobile phone panel in use. Based on the results of die flow analysis and drop simulation the optimal gate design scheme 2 is found, and the weld line position avoids the lower part of the elliptical hole, which is the weak part of the structure. The research results had certain reference significance for the optimization of injection process of plastic products.
\end{abstract}

\section{Keywords \\ Drop Simulation, Gate, Weld Lines}

\section{Introduction}

In the process of injection molding, there are many factors that affect the quality of products. Unreasonable factors such as product design, mold structure, molding process parameters, materials and injection equipment will lead to product defects. Reasonable use of mold flow analysis software can predict the 
possible defects in products and molds, solve problems in advance before mold manufacturing, reduce mold testing time, save time and reduce costs [1] [2]. With the rapid development of electronic products, people are using all kinds of electronic products in their lives. Many of the electronic products are made of plastic. Various defects such as weld mark, warpage, crack, silver wire, etc will appear in the molding process of plastic products. At the same time, it is inevitable for people to drop electronic products when using electronic products. When the plastic shell of electronic products collides with the ground, it often cracks. Therefore, it is necessary to combine the drop process analysis in the process of product injection molding analysis to improve the quality of products [3] [4] [5]. Today, with the increasing popularity of smartphones, traditional push-button mobile phones still have a large market, especially suitable for the elderly and children. In this paper, aiming at the problem of weld line defects in the injection process of a key phone panel, the injection process is optimized by using Moldflow and drop simulation. Through the optimization, the product defects are effectively solved, and the analysis process has a good reference for the comprehensive use of mold CAE software and drop simulation method to analyze the injection process of plastic parts.

\section{Modeling and Mesh Generation of the Mobile Phone Panel}

The 3D geometric model of mobile phone panel is designed in 3D software, as shown in Figure 1. The mobile phone panel model file in 3D software is saved as stl format file and transferred to Moldflow software. The size of mobile phone panel is $90 \mathrm{~mm} \times 40 \mathrm{~mm} \times 12 \mathrm{~mm}$, and the thickness is uniform, which is 2.5 $\mathrm{mm}$. The material of mobile phone panel is ABS, which has outstanding mechanical properties, certain chemical stability and good dielectric properties, good dimensional stability, easy forming and machining, and is suitable for making common mechanical parts, antifriction and wear-resistant parts and telecommunication structural parts.

The geometric model designed in 3D software needs to be imported into Moldflow for analysis and calculation. Firstly, the geometric model needs to be meshed, and automatic and manual meshing methods are combined. In the manual meshing method, the modification of aspect ratio is very difficult and needs to be carried out patiently and carefully. The aspect ratio of the detail part of the geometric model is often too large when meshing. Generally, the detail part of the structure is ignored as far as possible without affecting the product structure function, which can greatly reduce the workload of mesh modification. When the mobile phone panel is meshed, most of the meshes with large aspect ratio appear in the fillet around the model, as shown in Figure 2. In order to improve the accuracy of the model, the fillet is not removed here. In order to avoid changing the local shape of the model and improve the accuracy of the model, the method of local mesh encryption can be used when modifying the mesh of the fillet part. 


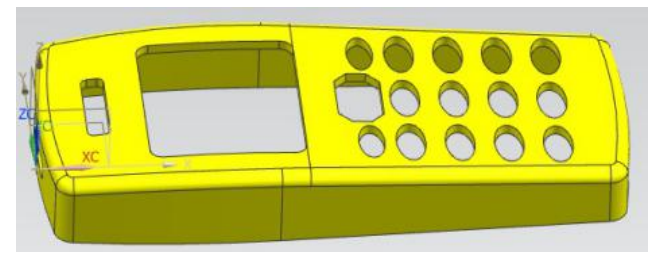

Figure 1. The geometric model of the mobile phone panel.

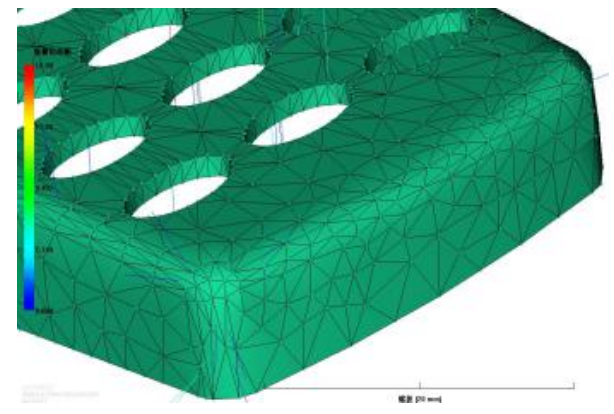

Figure 2. Local mesh generation.

The modified grid results are shown in Table 1 , and the grid quality meets the analysis requirements of injection molding.

\section{Gate Design of Mobile Phone Panel}

The choice of gate position directly affects the flow direction of the melt in the mold cavity, and thus affects the position of the weld line. Therefore, the reasonable choice of gate position is very important in the mold system design. Firstly, the optimal gate position of the mobile phone panel is analyzed, and a preliminary optimal gate setting scheme is found, which provides a reference gate position for further injection molding analysis.

The schematic diagram of the best gate for mobile phone is shown in Figure 3. The area in the ellipse is the best gate location area, and the gate setting in this area can ensure the balance of melt flow during injection. The final gate location also needs to consider the location of weld lines and mechanical properties.

According to the preliminary analysis results of the best gate, the initial design schemes of six gate positions are given, as shown in Figure 4. The distribution of weld lines after each flow analysis is shown in Figure 5.

The panel of the mobile phone adopts the traditional key mode. Multiple elliptical holes in the middle of the panel are pressed by fingers during use. The position under the elliptical hole is frequently stressed, which belongs to the weak part of the structure. When selecting the gate design scheme, it is necessary to consider that the weld mark should not appear in the weak part, otherwise, it is easy to break under the frequent action of external force. It can be seen from the weld trace distribution diagram of gate scheme shown in Figure 6 that the weld traces of scheme 1,3 and 4 all appear under the elliptical hole of the weak part of the structure, while the weld traces of scheme 2, 5 and 6 are located on the side of the elliptical hole, avoiding the weak part of the structure. 
Table 1. The mesh statistical chart of the mobile phone panel.

Number of triangular elements
Connected nodes
Connected area
Surface area/cm ${ }^{2}$
Aspect ratio
Edge detail
Orientation details
Intersection details
Matching percentage
matching=1.0 Best
00

Figure 3. The best gate of the mobile phone panel.
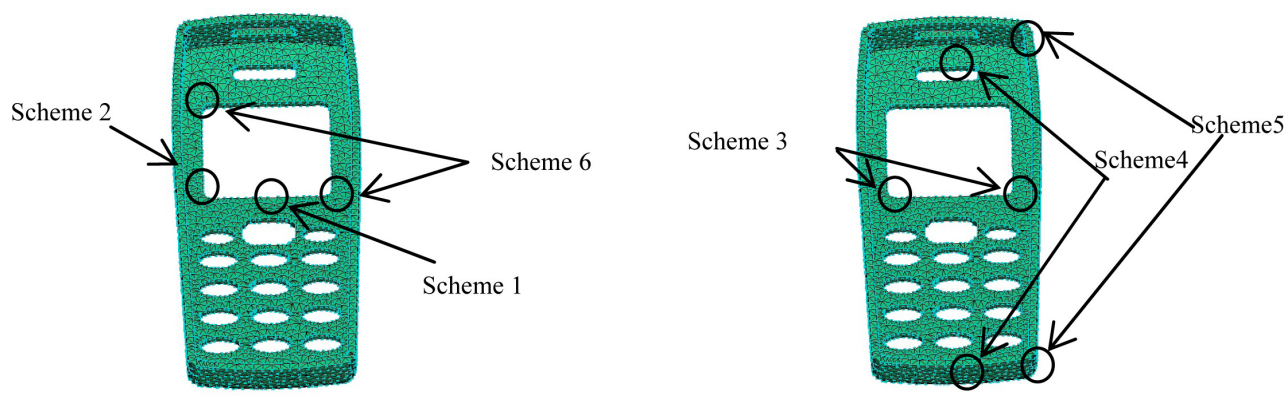

Figure 4. Design scheme of gate location.

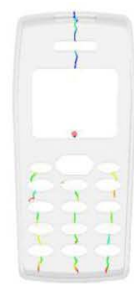

Scheme 1

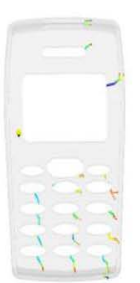

Scheme 2

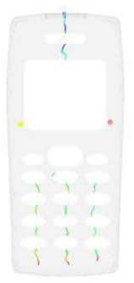

Scheme 3

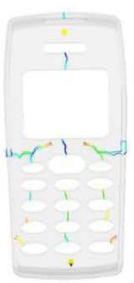

Scheme 4

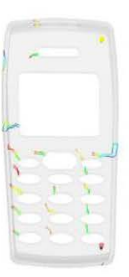

Scheme 5

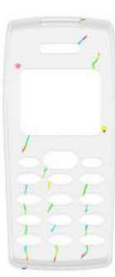

Scheme 6

Figure 5. Distribution of weld lines in six gate schemes. 


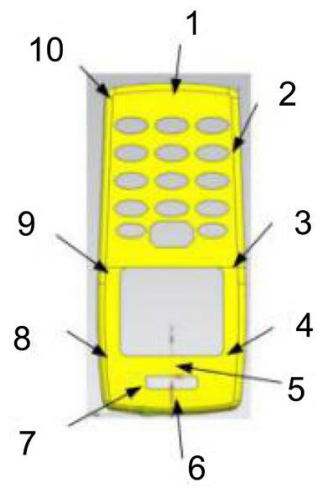

Figure 6. Position labeling of weld lines.

Furthermore, the flow and warpage analysis of the six gate schemes are carried out, and the mold flow analysis results of each gate scheme are obtained, as shown in Table 2.

According to the mold flow analysis results shown in Table 2, it can be seen that the maximum deformation of Scheme 1,2, 3 and 6 are relatively close and relatively small. According to the volume shrinkage, scheme 2 and scheme 3 are smaller. According to the filling time, scheme 3, 4 and 5 are smaller. From the point of view of the maximum residual pressure, scheme 2 and 3 are smaller.

In addition, the mobile phone, as a daily necessity, is easy to fall. When the mobile phone panel is impacted with the ground, if the maximum stress appears at the weld line, it is easy to crack. Therefore, it is necessary to analyze the drop stress state of the mobile phone panel, find the maximum stress position when the mobile phone panel is impacted with the ground, and the weld line position should avoid the maximum stress, it provides reference for gate location design.

\section{Drop Analysis of Mobile Phone Panel}

Using LS-DYNA display dynamics analysis software on ANSYS Workbench 15 finite element analysis platform, the drop process of mobile phone is simulated and analyzed. LS-DYNA display algorithm can be used to analyze various nonlinear structural impact dynamics problems, such as explosion, collision, metal deformation and other highly nonlinear problems. The main algorithm is Lagrange algorithm, with ale and Euler algorithm. It is mainly based on the explicit solution and has the function of implicit solution. It is mainly based on nonlinear analysis and has the function of static analysis. The powerful CAD bidirectional driving tool and meshing tool in ANSYS Workbench can quickly complete the definition of initial conditions and boundary conditions in LS-DYNA analysis.

The geometric model of mobile phone panel is designed in three-dimensional software and transmitted to the geometry module of workbench platform in a common data format. ABS is selected as the material of mobile phone panel and cement concrete as the ground material. The parameters of the two materials are shown in Table 3. 
Table 2. Mould flow analysis results of six gate schemes.

\begin{tabular}{cccccccc}
\hline & \multicolumn{2}{c}{ Scheme 1 } & \multicolumn{2}{c}{ Scheme 2 } & \multicolumn{2}{l}{ Scheme 3 } & \multicolumn{2}{l}{ Scheme 4 } & \multicolumn{2}{l}{ Scheme 5 } & Scheme 6 \\
\hline Maximum deformation/mm & 0.3689 & 0.3791 & 0.3387 & 0.3867 & 0.4045 & 0.3729 \\
Volume shrinkage/\% & 9.111 & 8.236 & 8.333 & 8.875 & 8.506 & 8.552 \\
filling time/s & 0.7 & 0.7139 & 0.5884 & 0.4628 & 0.5824 & 0.7067 \\
Maximum residual pressure/MPa & 50.42 & 47.19 & 47.78 & 51.18 & 50.35 & 50.31 \\
Main weld mark location & $1,5,6,10$ & $2,7,8$ & $1,5,6,10$ & $3,9,6$ & $2,3,7$ & $3,7,8$ \\
\hline
\end{tabular}

Table 3. Material parameters.

\begin{tabular}{ccccc}
\hline & Density $\left(\mathrm{kg} / \mathrm{m}^{3}\right)$ & $\begin{array}{c}\text { Elastic modulus } \\
(\mathrm{MPa})\end{array}$ & Poisson's ratio & $\begin{array}{c}\text { Shear modulus } \\
(\mathrm{MPa})\end{array}$ \\
\hline ABS material & 1020 & 1100 & 0.18 & 387 \\
Cement concrete materials & 2300 & 30,000 & 0.42 & 12,714 \\
\hline
\end{tabular}

According to the weld line distribution map of six gate schemes shown in Figure 5, mark the main weld line location on the mobile phone panel, as shown in Figure 6. The subsequent drop simulation mainly analyzes the stress distribution at the weld line location shown in Figure 6.

In order to shorten the simulation analysis time and reduce the distance between the mobile phone and the ground, the initial acceleration is set at the center of gravity of the mobile phone panel. The initial acceleration is calculated according to the formula $v_{0}=\sqrt{2 g h}$. According to the impact angle between the mobile phone and the ground, five typical ways are given, including head down vertical landing, bottom down vertical landing, side landing, head down 45-degree landing and bottom down 45-degree landing, as shown in Figure 7.

The stress values of 10 weld lines under five drop modes are given below, as shown in Table 4, and the maximum stress values of each weld line under five drop modes are given.

As can be seen from Table 4, the order of maximum stress at each weld mark is: Mark $1<\operatorname{mark} 9<\operatorname{mark} 5<\operatorname{mark} 2<\operatorname{mark} 7<\operatorname{mark} 4<\operatorname{mark} 8<\operatorname{mark} 3<$ mark $6<$ mark 10. The stress at weld mark 6 and mark 10 is relatively large. Scheme 1, 3 and 4 all contain these two marks. If these three schemes are adopted, the mobile phone will be easily damaged after falling. The stress of scheme 2, 5 and 6 is relatively small. When these three options are adopted, the mobile phone is not easy to be damaged after falling. Due to the limitation of the paper, the stress distribution of the mobile phone panel when the bottom is down 45-degree is given here, as shown in Figure 8. The colors in Figure 8 represent the stress distribution. It can be seen that the stress at the sharp corner of the lower end of the mobile phone is the largest.

According to the results of Moldflow analysis and LS-DYNA drop simulation analysis in ANSYS Workbench, it can be seen that the weld lines of option 2, 5 and 6 are located on the side of the elliptical hole, avoiding the weak link of the structure, and the maximum deformation of option 1, 2, 3 and 6 is relatively small. 
Table 4. Stress values at weld lines.

\begin{tabular}{cccccccccccc}
\hline & \multicolumn{1}{c}{ Stress at weld mark/MPa } \\
\cline { 2 - 10 } & Mark 1 & Mark 2 & Mark 3 & Mark 4 & Mark 5 & Mark 6 & Mark 7 & Mark 8 & Mark 9 & Mark 10 \\
\hline Head down vertical landing & 0.8371 & 1.409 & 1.482 & 2.137 & 1.157 & 14.113 & 1.042 & 0.809 & 1.151 & 3.943 \\
Bottom down vertical landing & 1.459 & 2.105 & 5.601 & 1.375 & 2.047 & 7.706 & 1.001 & 1.161 & 2.511 & 7.819 \\
Side landing & 2.135 & 1.539 & 2.639 & 2.094 & 2.452 & 1.129 & 1.183 & 2.015 & 2.011 & 17.119 \\
head down 45-degree landing & 0.791 & 2.068 & 2.932 & 3.687 & 2.421 & 1.576 & 3.383 & 5.146 & 1.281 & 1.623 \\
bottom down 45-degree landing & 2.068 & 2.523 & 1.677 & 2.915 & 0.957 & 0.951 & 1.822 & 1.196 & 1.437 & 12.368 \\
Maximum stress & 2.135 & 2.523 & 5.601 & 3.678 & 2.452 & 14.113 & 3.383 & 5.146 & 2.511 & 17.119 \\
\hline
\end{tabular}

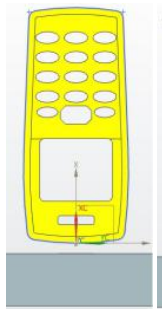

(a)

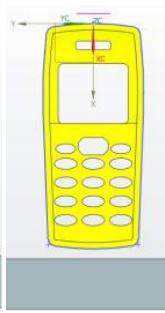

(b)

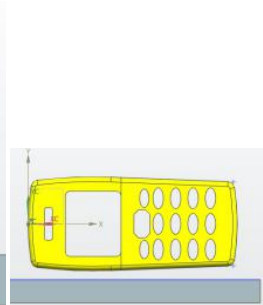

(c)

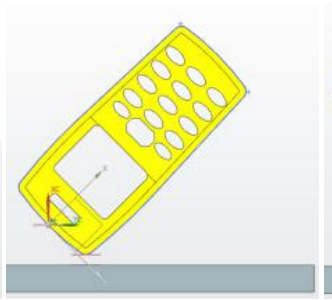

(d)

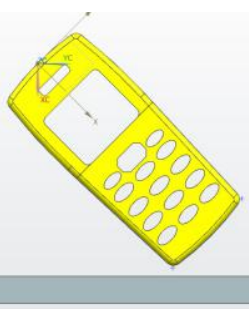

(e)

Figure 7. Drop mode of the mobile phone panel. (a) Head down vertical landing; (b) Bottom down vertical landing; (c) Side landing; (d) head down 45-degree landing; (e) bottom down 45-degree landing.
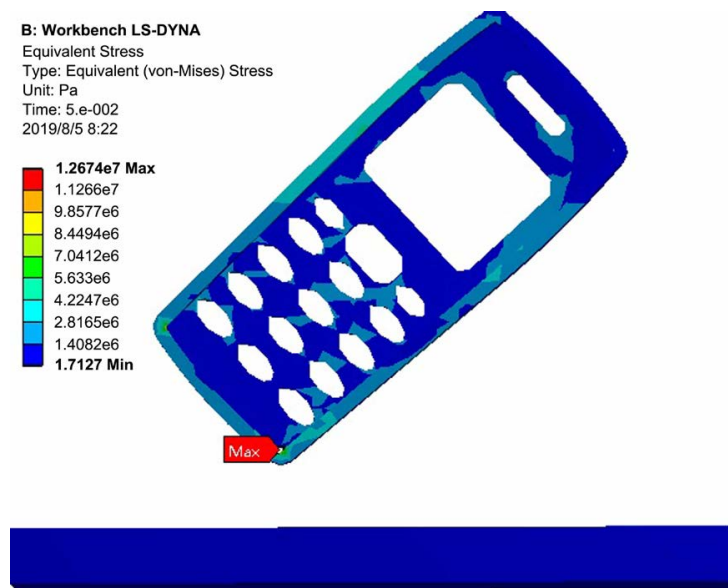

Figure 8. Stress distribution of the mobile phone panel.

According to the volume shrinkage, scheme 2 and scheme 3 are smaller. According to the filling time, scheme 3,4 and 5 are smaller. From the point of view of the maximum residual pressure, scheme 2 and 3 are smaller. The stress of scheme 2, 5 and 6 is relatively small. When these three schemes are adopted, the mobile phone is not easy to be damaged after falling. So scheme 2 is better.

\section{Conclusions}

1) The high quality mesh model of mobile phone panel is established in 
Moldflow, and six kinds of gate design options are established. The distribution position, maximum deformation, volume shrinkage, filling time and maximum residual stress of weld lines under each gate option are analyzed. According to the analysis, it is concluded that the option 1 and 6 with smaller deformation can be avoided, and the scheme 2 and 6 with smaller deformation can be avoided. According to the volume shrinkage, scheme 2 and scheme 3 are smaller. According to the filling time, scheme 3,4 and 5 are smaller. From the point of view of the maximum residual pressure, scheme 2 and 3 are smaller.

2) Through the LS-DYNA drop simulation analysis in ANSYS Workbench, it can be concluded that the stress of scheme 2,5 and 6 is relatively small. With these three options, the mobile phone is not easy to break after landing.

3) Based on the results of die flow analysis and drop simulation, it can be concluded that scheme 2 is better, the weld line position avoids the lower part of the elliptical hole, which is the weak part of the structure. The results of maximum deformation, volume shrinkage, filling time and maximum residual stress are better, and the stress at the weld line is smaller after the drop of mobile phone.

\section{Acknowledgements}

This work supported by Suzhou science and technology planning project (SYG201823); Sunzhou vocational university research project (SVU2018YY07); sponsored by Power and ultrasound research center of Suzhou Vocational University

\section{Conflicts of Interest}

The authors declare no conflicts of interest regarding the publication of this paper.

\section{References}

[1] Wang, G. and Shan, Y. (2007) Application Example of Moldflow Mold Analysis. Tsinghua University Press, Beijing, 243-244.

[2] Du, J. (2018) Optimization Analysis of a Soap Box Combined Cavity Flow Balance Based on Moldflow. China Plastics Industry, 46, 72-76.

[3] Xiong, J.-Y. and Xin, Y. (2003) Application of Drop Simulation in Injuction Molding. Journal of Plasticity Engineering, 10, 66-69.

[4] Ding, J.-B. and Cao, J.-D. (2012) Optimization of Injection Mobile Phone Shell Facing Drop Simulation. Plastics, 41, 31-33.

[5] Feng, Q.H., Zha, W.S. and Peng, B.Y. (2012) Optimization of Injection Molding Molding for Drop Simulation and Destruction. Engineering Plastics Application, 41, 31-33. 